\title{
Investigation of Influence of Oxygen on Process of Whiskey Ripening in New Design of Extractor
}

\author{
D.M. Borodulin \\ Doctor of Technical Sciences, Head of the Department \\ technological design of food production \\ Kemerovo State University \\ Kemerovo, Russia Federation \\ borodulin_dmitri@list.ru
}

\author{
A.N. Potapov \\ Candidate of Technical Sciences, Department \\ technological design of food production \\ Kemerovo State University \\ Kemerovo, Russia Federation \\ potap-1961@list.ru
}

\author{
M.V. Prosin \\ Candidate of Technical Sciences, Department \\ of Technosphere Safety \\ Kemerovo State University \\ Kemerovo, Russia Federation \\ prosinmv@yandex.ru
}

\begin{abstract}
The results of investigations of extraction of oak chips by $50 \%$ aqueous-alcoholic solution during the ripening of the viscous beverage are presented. Studies were carried out in an extractor of the original design using oak chips "Sweet Vanilla". When extracting chips, the gas mixture with oxygen extractor, extraction, oak chips, whiskey, optical density, polyphenols, dry substances was periodically treated. The results of determining the optical density, the content of polyphenols and dry substances in the extract for $\mathbf{3 5 0}$ days of aging are obtained. As a result of the analysis of the obtained data, rational parameters of the ripening process of the viscous beverage are determined. The results of the investigations testify to the intensification of the process of obtaining whiskey during periodic saturation of the system of interacting phases with an oxygen medium.
\end{abstract}

Keywords - extractor, extraction, oak chips, whiskey, optical density, polyphenols, dry substances

\section{INTRODUCTION}

In the world market of alcoholic beverages there is a whole range of alcoholic beverages (vodka, whiskey, gin, sake), which are produced from grain raw materials. Of all the variety of drinks, whiskey stands out - one of the most popular strong alcoholic beverages in the world. This drink has unique flavor and aroma tones. Whiskey is produced by aging a distillate (alcohol-whiskey), obtained from grain raw material after a certain processing with oak wood. The variety of methods of production of this drink explains a rather large number of names for brands of whiskey. In different countries there are historically developed technological and tasting traditions. Currently, there are five main types of whiskey: Scottish, Irish, American, Canadian and Japanese.
The traditional tastes of the population of Russia are focused on strong drinks made from alcohol derived from wheat. At the same time, the analysis of the Russian market of spirits shows that the tastes of the Russian consumer are gradually changing. Currently, more than $40 \%$ of Russians prefer such drinks as cognac and whiskey, instead of vodka and strong tinctures. Also, most consumers buy "premium" class drinks [1-3].

It should be noted that until 2015 in Russia there were standards only for the production of distillates from grapes to produce cognacs and brandy. The State Standard "Grain Distillate. Specifications" has come into force since July 1, 2015. It allows the production of whiskey in Russia.

The traditional method of obtaining whiskey can be divided into five main stages: the preparation of fermented wort from cereals; production of alcohol-whiskey; distillate aging with oak wood for a certain time; preparation and processing of samples of the drink; bottling and packaging of finished products.

To obtain the wort, the ground malt is mixed with hot water. Further, yeast is added to the cooled mixture, fermentation is carried out for 48 hours, after which a low alcohol drink of about 5\% strength is obtained. The resulting beverage is subjected to two-, three-stage distillation. Next, alcoholwhiskey is poured into oak barrels and left to ripen for a period of at least 3 years. The finished product is subjected to cleaning, blending and bottling in a container. It should be noted that the parameters of the finished beverage are significantly influenced by the properties of the grain, as well as the process of obtaining and drying malt. The temperature conditions of barley germination, the equipment used and the technological conditions for obtaining malt, affect not only quantitative indicators in the preparation of wort, but also the organoleptic characteristics of the finished product.

The main stage of obtaining whiskey is certainly the distillate extract. In its essence, this is an extraction process 
during which certain components pass from the solid phase to the liquid one. It is this stage that largely determines the final taste, color and aroma of the resulting beverage. Oak wood enriches alcohol with products of ethanolysis and lignin hydrolysis, polysaccharides, tannins and other components that, under the influence of oxygen, are converted into substances that determine the quality of the final product. At the same time, this technological operation assumes a long aging of spirits in expensive oak barrels. The duration of aging of alcohols to a large extent determines the cost of the resulting beverage. Therefore, reducing the holding time is the most important task, which allows increasing the productivity and reducing the cost of the final product. This goal is justified, if there is no loss of quality characteristics of whiskey.

To reduce the aging time of beverages, the following methods of intensifying the extraction process are proposed [411]:

1) Extraction by electric discharges. Under the influence of an electric discharge, shock waves form in the extracted mixture. Under their influence there is an intensive mixing of the mixture, the diffusion boundary layer almost completely disappears. The appearance of shock waves facilitates the penetration of the extractant into cells, where rapid intracellular diffusion proceeds. In addition, as a result of a spark discharge, plasma cavities are formed in the liquid, which, expanding, reach a maximum volume and collapse, which leads to the destruction of cellular structures and the leaching of substances from cells. This way of intensification of the process is constructively complex and involves a considerable consumption of electricity. This method cannot be applied with the traditional infusion of alcohol-whiskey in oak barrels, because the intensification of the process with this method is possible when suspended solids are present in the bulk of the extractant.

2) Extraction using ultrasound effect. Practical studies have shown that the use of ultrasound in the extraction process leads to its significant intensification by increasing the active contact surface of solid particles and the liquid phase. Also, when the ultrasound is applied, the hydrodynamic situation at the interface is improved. The results of the studies show that ultrasound makes it possible both to increase the mass-transfer coefficient and to reduce the time required to obtain a given technological result during extraction for several times. Ultrasonic devices for extraction processes have a fairly simple design. The main reason for curbing the active use of these devices in industry is their low productivity due to the need for a sufficiently long-term exposure during extraction.

3) Extraction in a gaseous medium with predominance of oxygen (ozone). An important step in obtaining a rich taste and aroma in the production of alcoholic beverages such as whiskey is contact with oxygen. Some scientists suggest that instead of the oxygen medium, one can use a mixture saturated with the oxygen isotope - ozone. In the study of the effect of the oxygen medium, it was found that this extraction method can reduce the "aging" time of whiskey. It was noted that the content of methanol increases with the increasing contact time with the gaseous medium. The prolonged oxygen exposure to extracted materials leads, on the one hand, to accelerating the beverage ripening, on the other hand - to the deterioration in the organoleptic properties, such as the taste and aroma of the viscous beverage.

The aim of this work is to develop and study a new design of an extractor for a solid-liquid system in the presence of a gaseous medium, which significantly reduces the time of ripening of whiskey without loss of quality characteristics (organoleptic properties).

To achieve the goal, the following tasks are set:

1. study of the classical way of whiskey ripening;

2. development and research of a new extractor design for the solid-liquid system;

3. determination of rational parameters of the whiskey ripening process when it is saturated with oxygen in a new apparatus;

4. determination of the basic parameters of the whiskey obtained.

\section{OBJECTS AND METHODS OF RESEARCH}

The research was carried out in the laboratory conditions of the department of Technological design of food production at the Kemerovo State University.

The object of the study is the extractor of a new structure for extracting the required components from oak chips [12]. Its design allows one, during the extraction process, to oxygenate the mixture of water-alcohol solution with chips, thereby accelerating the ripening process of the drink (Fig. 1).

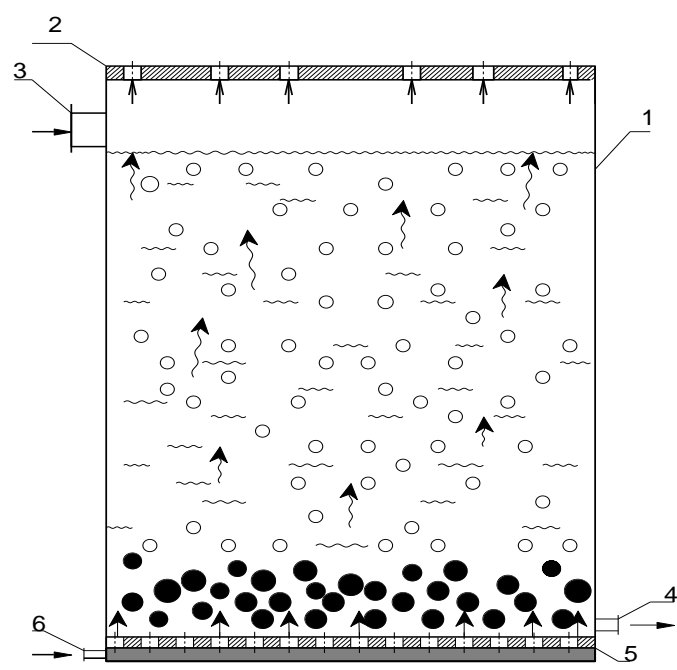

Fig. 1. The extractor of batch action: 1 - cylindrical body; 2 - perforated flat cover; 3 - nozzle for feeding the extractant and solid phase; 4 - nozzle for the exit of the finished product; 5 - gas distribution device; 6 - nozzle for oxygen supply

The work of the new extractor is carried out as follows. The solid phase and water-alcohol solution are loaded into the extractor through the feeding nozzle 3, evenly filling its working volume. To increase the intensification of the 
extraction process under pressure, oxygen is fed to the lower part of the apparatus through the supplying nozzle 6 into the gas distribution device 5 . The oxygen bubbles formed in it, rising from the bottom up, lead to the fluidized state of oak chips, chaotically mixing it in the entire volume of the liquid. This activates the extraction process by updating the contact surface of the phases. In addition, the introduction of oxygen into the mixture of a water-alcohol solution with oak chips leads to oxidative transformations in the system, which accelerates the ripening process of the drink. Oxygen, which has passed through the entire volume of the extractant, is removed from the upper part of the apparatus through the holes located in the cover 2, which subsequently overlap to prevent the alcohol solution from evaporating into the environment after the oxygen supply has ended. At the end of the whole process, the final liquid product is removed from the lower part of the cylindrical body 1 , discharging nozzle 4 . The waste oak chips are removed from the apparatus through its upper part after removing the cover 2 .

Intensification of the extraction process of the required components from oak chips is achieved due to the uniform introduction of oxygen into the extractor, which leads to the creation of a bubbling regime in the system and as a consequence to the repeated movement of solid particles in the entire volume of the water-alcohol mixture. The introduction of oxygen leads to the oxidation of the propylphenol components of lignin in a double bond to form the simplest aromatic aldehydes such as vanillin, as well as to oxidation of tanning substances of oak chips with the formation of soluble products that improve the organoleptic characteristics of the finished extract.

The subject of the study is the determination of physicochemical parameters of extracts from oak chips, the study of the kinetics of extraction of components with a $50 \%$ aqueous alcohol solution, the identification of rational technological parameters affecting the extraction rate.

In the study the following was used:

1) Alcohol: ethyl alcohol of "Luxe" class meets the requirements of State Standard 5962-2013. Lux is a highquality grain ethyl alcohol obtained by rectification from wheat, barley, rye or a mixture of grains. Most of the vodka and liquor and vodka products of the Russian production are made from alcohol of the "Lux" class. It is a clear, colorless liquid, free of foreign smells and flavors. The content of ethyl alcohol is $96.5 \%$.

2) Water: the water used in the studies meets the requirements of State Standard 6709-72 "Distilled water. Technical conditions (with Changes No. 1, 2)"

3) Oak chips: for the experiments, oak chips of the "Sweet Vanilla" brand were used. Wood chips are an economical alternative to a French or Serbian oak barrel. Oak chips pass through the roasting stage in a special oven without the use of any chemicals. The wood selected for the production of this wood chips is supplied by Tonnellerie Radoux, one of the best cooperage productions in France. Before further processing of chips, the process of loosening is carried out within 16-24 months. Chips are small particles of wood 2.0-2.5 cm long, up to $1 \mathrm{~cm}$ wide and 2-3 mm thick. The color of the wood is from light brown to saturated dark brown depending on the brand. In the chips of the brand "Sweet Vanilla", light brown tones predominate. These chips add a balanced vanilla tone to the drink and allow you to achieve a noble taste and light sweetness aftertaste [13-14].

\section{A. Methods of work:}

At the beginning of the work samples (three replicates for each type of experiment) of water-alcohol solutions with an alcohol concentration of $50 \%$ were prepared. In each sample, oak chips were added in a ratio of $1 \mathrm{~g}$ chips to $500 \mathrm{ml}$ solution. Prepared samples were divided into 4 groups. One group was maintained in a classical manner, thereby obtaining a control sample. The remaining 3 groups of samples were exposed to oxygen in a batch extractor with different duration, namely: once in 2 days the samples were saturated with oxygen for 5 , 10,15 minutes, respectively. The duration of the experiment was 350 days.

To evaluate the results of processes occurring when the oak chips are kept in water-alcohol solution, the following measurement methods were applied [15-17]:

1. Determination of the optical density by the spectrophotometric method.

2. Determination of the content of polyphenols by the Yerumanis method.

3. Determination of the dry matter content by the drying method.

The color index of the resulting beverage during its aging by the extraction of oak wood in a water-alcohol solution was studied using the spectrophotometric method [18]. The nature of the change in the color of the drink was determined on a photoelectric colorimeter. When determining the density, $1 \mathrm{~cm}$ thick cuvettes were used. The wavelength was $540 \mathrm{~nm}$. The readings of the photoelectric colorimeter were measured with respect to a $50 \%$ aqueous alcoholic solution of ethyl alcohol. The absorbance of the samples was measured every 25 days for the entire duration of the experiment.

Aromatic characteristics of the drink were evaluated by the content of polyphenols by the method of Yerumanis. This method is based on the reaction of polyphenols with trivalent iron salts in an alkaline medium. Three reagent samples were prepared for determination. For the first sample, $10 \mathrm{ml}$ of extract and $8 \mathrm{ml}$ of a previously prepared carboxymethyl cellulose solution (CMC) were added to a $25 \mathrm{ml}$ volumetric flask, $0.5 \mathrm{ml}$ of an iron reagent was added, $0.5 \mathrm{ml}$ of ammonia solution was added and the solution was adjusted to the mark with distilled water. After 10 minutes, the optical density was measured on a photoelectric calorimeter (at a wavelength of 600 $\mathrm{nm}$ ) in a cuvette of $10 \mathrm{ml}$, against distilled water. For the second sample, $10 \mathrm{ml}$ of extract and $8 \mathrm{ml}$ of CMC were added to a 25 $\mathrm{ml}$ volumetric flask, $0.5 \mathrm{ml}$ of ammonia solution was added and adjusted to the mark with distilled water, after 10 minutes the optical density was measured similarly to the first sample. For the third sample, a $25 \mathrm{ml}$ flask was also used in which $10 \mathrm{ml}$ of distilled water, $8 \mathrm{ml}$ of CMC solution, and $0.5 \mathrm{ml}$ of ammonia solution were added. After bringing the volume to the mark (10 
minutes), the optical density was determined as in previous cases.

The contents of the dry extractive substances in the wateralcohol solution with the chips were determined by the drying method. By quantitative indices of concentration, one can judge the taste characteristics of the obtained beverage samples. To determine the dry substances on an analytical scale, empty bucks were weighed to an accuracy of $0.0001 \mathrm{~g}$, into which 5 $\mathrm{cm}^{3}$ of a water-alcohol solution was pipetted. The bucks with the solution were placed in a drying cabinet at a temperature of (90-95) ${ }^{\circ} \mathrm{C}$ and dried for two hours. After cooling, the weight of the buck with a dry residue was determined.

\section{RESULTS AND DISCUSSION}

Analysis of beverage samples was obtained with the use of "Sweet Vanilla" chips.

The optical density of samples with chips "Sweet Vanilla" from the time of the experiment for different duration of oxygen saturation of the control samples is shown in Fig. 2. The same graph shows the change in the optical density of the control sample (without saturation of the system with oxygen).

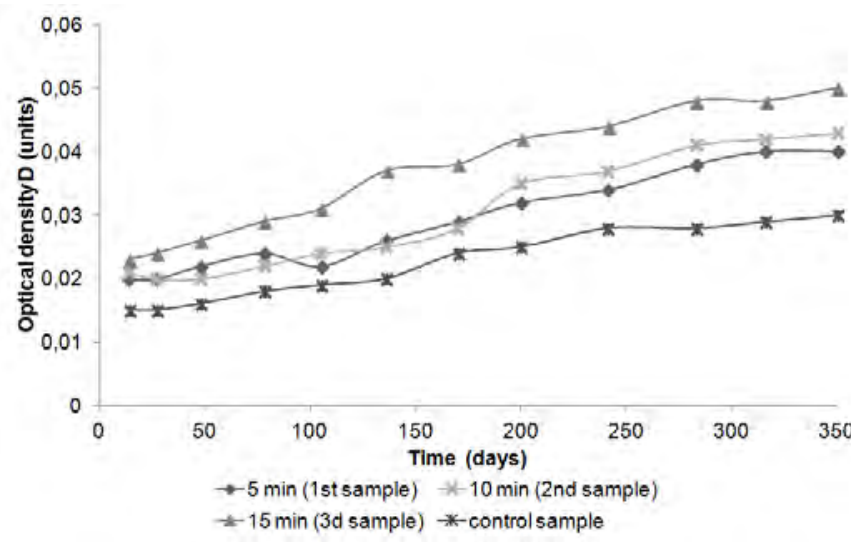

Fig. 2. Dependence of the optical density of the samples on the chips, "Sweet vanilla" on the time of ripening of the drink for different saturation duration of the samples with oxygen

Figure 2 shows that with an increase in the duration of the extraction process, the optical density of the beverage samples is expected to increase. Also, the optical density increases with increasing time of saturation of the mixture with oxygen in relation to the control sample.

At the initial stage of the process (after 25 days), the optical density of control sample D was 0.015 units, and after expiration of 325 days - 0.03 units. A sufficiently sharp extraction of substances that determine the color characteristics of the drink at the initial stage of extraction is due to the transfer of components from the surface layers of the solid phase. The active absorption of the extractant by the particles of the dry solid phase and the dissolution processes associated with this phenomenon of easily accessible components located deep in the particles of oak chips also takes place here. In the future this process slows down and within 300 days the same number of components is extracted, as for the first 25 days of extraction.
The subsequent transfer of matter from the inner layers of the solid phase becomes rather difficult.

When the beverage samples are saturated with oxygen for a certain time, an increased yield of substances determining the optical density is observed. The maximum value of optical density $\mathrm{D}$ is achieved with a 15-minute exposure to oxygen on the sample. At the same time this indicator has changed from 0.023 to 0.05 units. A higher level of recovery is due to the fact that oxygen bubbles passing through the apparatus from the bottom up create a fluidized state in the system involving oak chips in the chaotic motion throughout the volume of the wateralcohol mixture, thereby intensifying the extraction of the target components.

Comparing the obtained final numerical values of the optical density of the control and the third samples, it can be noted that exposure to oxygen for 15 minutes leads to an increase in the optical density almost 2 times. Therefore, it can be concluded that this method allows the process of ripening of the drink to be accelerated. Further increase in the duration of treatment with oxygen is impractical. With prolonged contact of oxygen with this system, compounds that degrade the quality of the finished product may appear. Thus, an increase in the content of lignin increases the acidity of the drink, which is unusual for a whiskey drink and worsens the taste of the finished product.

The content of polyphenols in the samples using "Sweet vanilla" chips after the system is saturated with oxygen after 350 days of maturing of the drink is shown in Fig. 3

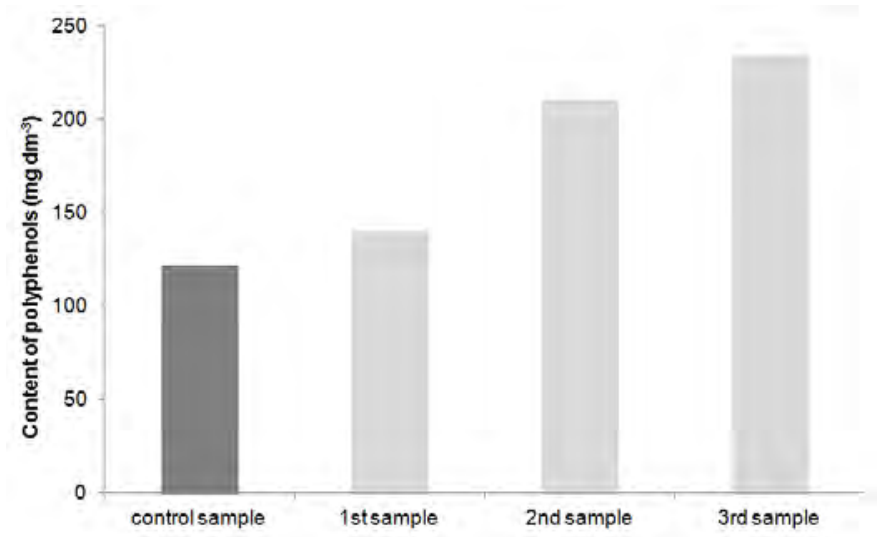

Fig. 3. The content of polyphenols in samples with "Sweet Vanilla" chips after saturation with oxygen after one year of soaking

It can be noted that in a sample maintained in the classical way, the content of polyphenols is $121.8 \mathrm{mg} \mathrm{dm}^{-3}$. Based on the results obtained, it follows that oxygen saturation of the samples favorably affects the recovery of polyphenolic substances from oak chips. In all three samples an increase in the yield of polyphenol is noted. Its maximum amount is observed in samples with 15-minute oxygen saturation and is $234 \mathrm{mg} \mathrm{dm}^{-3}$, which almost double the control sample. 
This also indicates a positive effect of oxygen on the ripening process of the drink. The increase in the yield of polyphenols in the oxygen saturation of the system can be explained not only by improving the hydrodynamic situation in the apparatus, but by oxidative transformations in the mixture.

The results on the content of dry extractive substances in the samples after a year are shown in Fig. 4.

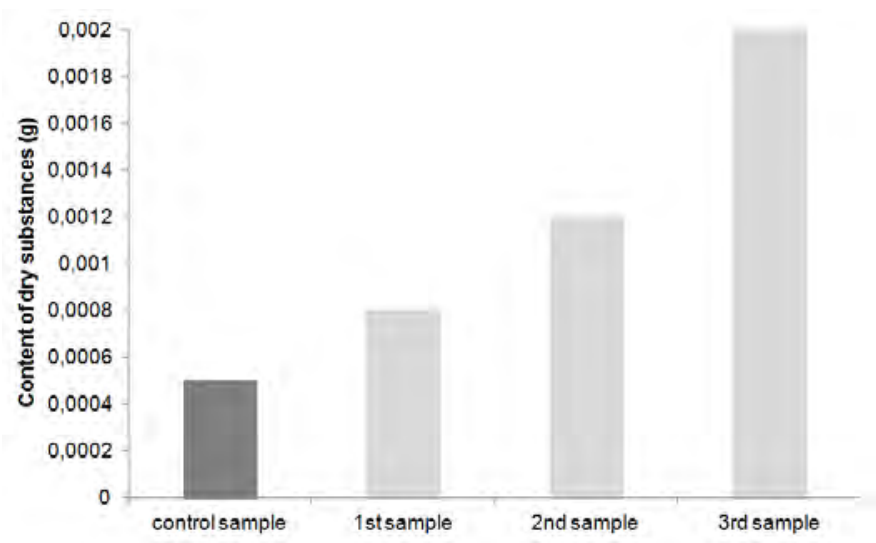

Fig. 4. The content of dry extractive substances in samples with "Sweet vanilla" chips after saturation with oxygen after one year of exposure

It can be seen from Fig. 4 that an increase in the yield of dry substances in all three samples is observed with respect to the control one. As the time of saturation of the sample with oxygen increases, an increasing tendency is also observed. In the control sample, the solids content is 0.0005 grams, and in the third sample 0.002 grams. This indicates a positive dynamics of the effect of oxygen on the yield of dry substances. The content of dry substances in the beverage is increased 4 times during the period under study. Somewhat lower is the content of solids in five- and ten-minute treatment. Such results can be explained by the intensity of mixing of solid particles in the liquid as the gas passes through the extractor, which is determined by the saturation duration of the system with oxygen.

Based on the results obtained, it can be concluded that the oxygen saturation of the system is promising when extracting oak chips with a $50 \%$ hydroalcoholic solution in the developed extractor. The results of studies of ready-to-drink drinks, which were carried out according to three indices, testify to the advisability of using 15 minutes of oxygen treatment of the system under study.

At the final stage of the studies to determine the content of volatile organic compounds, the samples studied after preliminary distillation, were analyzed by chromatographymass spectrometry.

The results of chromatographic analysis of a sample of a beverage obtained with a 15 minute oxygen saturation of the system are shown in Fig. 5.

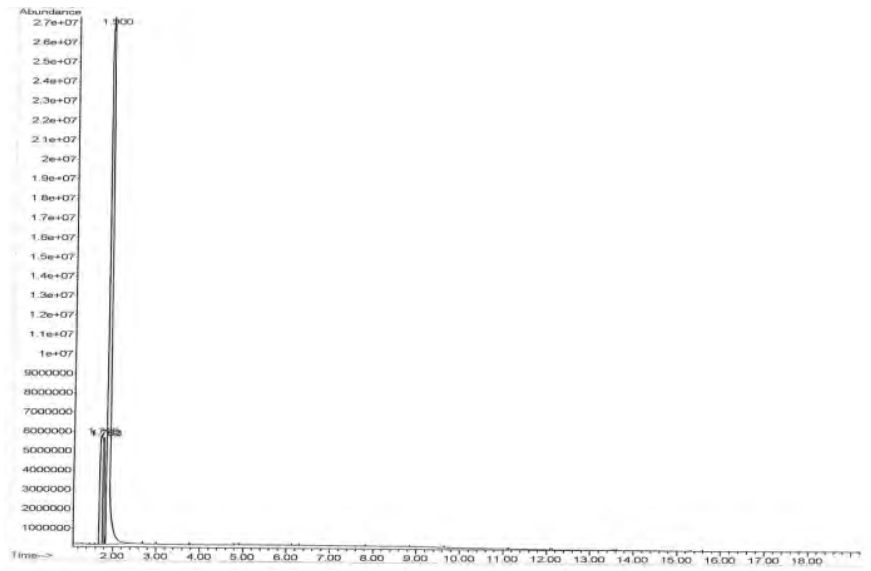

Fig. 5. Chromatogram of the sample drink under 15 minutes saturation of the system with oxygen

The chromatograms of the samples with a different oxygen saturation time and a control sample are similar to the example shown in Fig.5.

The chromatograms present peaks characteristic only for the content of ethanol and water. The first peak shows $30 \%$ water content, and the second $-70 \%$ alcohol in the finished product. Thus, it can be concluded that saturation with oxygen under these conditions does not lead to the oxidation of ethanol to harmful volatile products. Therefore, this method can be used to accelerate the ripening of whiskey without harm and changing the quality indicators.

\section{IV.CONCLUSION}

According to the results of the research, the following conclusions can be drawn:

A study of the process of obtaining whiskey using oak chips "Sugar Vanilla" was carried out.

A device for carrying out the extraction process in a solidliquid system in the presence of a gas (oxygen) medium was developed. The design of the apparatus allows for periodic saturation of the system with a gaseous medium without loss of the volatile solvent during further extraction.

The rational regimes of the process of obtaining whiskey in the developed apparatus are determined - the duration of the oxygen saturation of the system is 15 minutes, the processing frequency is once in two days, the aging time is not less than 360 days.

Laboratory tests showed that the beverages obtained during the experiments had higher optical density, polyphenols and dry substances content with respect to the control sample. Chromatographic analysis indicates that there are no undesirable compounds in the samples obtained.

In general, it can be concluded that the oxygen saturation is promising in the process of extracting oak chips with $50 \%$ aqueous-alcoholic solution. The duration of the production process of whiskey ripening can be reduced at least 2 times. 


\section{References}

[1] S.Ž. Gorjanović, D.Ž. Sužnjević, M.M. Novaković, P.V. Vukosavljević, F.T. Pastor, and V.V. Tešević, "Polarographic assay based on hydrogen peroxide scavenging in determination of antioxidant activity of strong alcohol beverages," Journal of agricultural and food chemistry, Vol. 58, no 14,2010 , pp. 8400-8406.

[2] M.A. Jochmann, D. Steinmann, S. Manuel, and T.C. Schmidt, "Flow injection analysis-isotope ratio mass spectrometry for bulk carbon stable isotope analysis of alcoholic beverages," Journal of agricultural and food chemistry, Vol. 57, no 22, 2009, pp. 10489-10496.

[3] K. Linderborg, M. Salaspuro, and S. Väkeväinen, "A single sip of a strong alcoholic beverage causes exposure to carcinogenic concentrations of acetaldehyde in the oral cavity," Food and chemical toxicology, Vol. 49, no 9, 2011, pp. 2103-2106.

[4] M.L.M.N. Cerutti, A.A.U. De Souza, and S.M.D.A.G.U. De Souza, "Solvent extraction of vegetable oils: numerical and experimental study," Food and bioproducts processing: transactions of the institution of chemical engineers, part C. Vol. 90, no 2, 2012, pp. 199-204.

[5] M.D. Esclapez, J.V. García-Pérez, A. Mulet, and J.A. Cárcel, "Ultrasound-assisted extraction of natural products," Food engineering reviews, Vol. 3, no 2, 2011, pp. 108-120.

[6] K.V. Loginova, E. Vorobiev, O. Bals, and N.I. Lebovka, "Pilot study of countercurrent cold and mild heat extraction of sugar from sugar beets, assisted by pulsed electric fields," Journal of food engineering, Vol. 102, no 4, 2011, pp. 340-347.

[7] D.L. Luthria, "Optimization of extraction of phenolic acids from a vegetable waste product using a pressurized liquid extractor," Journal of functional foods, Vol. 4, no 4, 2012, pp. 842-850.

[8] O. Parniakov, N.I. Lebovka, E. Van Hecke, and E. Vorobiev, "Pulsed electric field assisted pressure extraction and solvent extraction from mushroom (agaricus bisporus), "Food and bioprocess technology, Vol. 7, no 1, 2014, pp. 174-183.

[9] A.N. Shikov, O.N. Pozharitskaya, V.G. Makarov, and M.N. Makarova, "New technology for preparation of herbal extracts and soft halal capsules on its base," American-Eurasian journal of sustainable agriculture, Vol. 3, no 2, 2009, pp. 130-134.
[10] Y. Xu, Y. Bailina, Z. Ge, T. Ding, X. Ye, and D. Liu, "Effects of ultrasound and/or heating on the extraction of pectin from grapefruit peel," Journal of food engineering, no 126, 2014, pp. 72-81.

[11] Y. Tao, and D.W. Sun, "Enhancement of food processes by ultrasound: a review," Critical reviews in food science and nutrition, Vol. 55, no 4, 2015, pp. 570-594.

[12] D.M. Borodulin., E.A. Vechtomova, A. A. Fajner, and K. V. Klopova, "Extractor of batch action for extraction of target components from oak chips," Patent RF, no 2644914, 2018.

[13] C. Pizarro, S. Rodríguez-Tecedor, I. Esteban-Díez, N. Pérez-Del-Notario, and J.M. González-Sáiz, "Experimental design approach to evaluate the impact of oak chips and micro-oxygenation on the volatile profile of red wines," Food chemistry, no 148, 2014, pp. 357-366.

[14] Y. Tao, D.-W. Sun, A. Górecki, W. Błaszczak, G. Lamparski, and R. Amarowicz, "A preliminary study about the influence of high hydrostatic pressure processing in parallel with oak chip maceration on the physicochemical and sensory properties of a young red wine," Food chemistry, no 194, 2016, pp. 545-554.

[15] O. Noréus, and A. Trigell, "Measurement of terrain values and drawbar pull for six wheeled vehicle on sand," 16th International Conference of the International Society for Terrain Vehicle Systems. ISTVS, Turin, 2008, pp. 250-257 (in Italian).

[16] M.A.L. Oliveira, M. Heller, L. Vitali, G.A. Micke, and A.C.O. Costa, "A rapid sample screening method for authenticity control of whiskey using capillary electrophoresis with online preconcentration," Journal of agricultural and food chemistry, Vol. 57, no 22, 2011, pp. 6882-6888

[17] S.A. Sukhikh, "Technology of alcohol oxidase production from yeast candida boidinii for use in functional foods intended for withdrawa syndrome alleviation," Foods and raw materials. Vol. 2, no 2, 2014, pp. 53-58.

T.S. Collins, S.E. Ebeler, and J. Zweigenbaum, "Profiling of nonvolatiles in whiskeys using ultra high pressure liquid chromatography quadrupole time-of-flight mass spectrometry (uhplc-qtof ms)," Food chemistry, Vol. 163, 2014, pp. 186-196. 